\title{
The Research and Development of Integrated Operation-Maintenance Simulation Training System
}

\author{
Feng Gao' ${ }^{1}$ Xiue Zhang' ${ }^{2}$ Lianzheng Zhao ${ }^{3}$ \\ ${ }^{1}$ Beijing Kedong Electric Control System Co. Ltd., Beijing, China \\ ${ }^{2}$ Datong Electric Power Senior Technical School, Datong, China \\ ${ }^{3}$ Jibei Electric Power Training Center, Beijing, China \\ Email: YL 10000@sina.com
}

Received September 2013

\begin{abstract}
With deep development of state grid's system of "Three Sets of Five [1]", China is in urgent need of establishing an appropriate type of simulation system to rapidly improve operation efficiency and the level of maintainers, which aim at the integrated operation of substation operation and maintenance service. This article gives an introduction of a simulation training system which is designed for operation-skills training in electrical systems. By the composition of the multiple subjects and skills training for operations staff, this system can provide human guarantee and intellectual support for the "Big-Centralized Overhal".
\end{abstract}

\section{Keywords}

\section{Substation; Operation and Maintenance; Integration; The Simulation Training}

\section{Introduction}

The State Grid Corporation of China Worker's congress proposed: Building a modern company shall focus on the establishment of management system. One of the most important components of the "Five integration" is integrated recondition, it deepens the integration revolution and will build up a matrix production management system on aspects such as management model study, production process optimization, lean production management and perfected standard system. The revolution of management system brings up an improved requirement of operation-maintenance (OM) [2] staff, the training of OM staff is the key to the success of integrated recondition model [3].

Electrical power system simulation research is firstly operated on grid simulation research, the power grid yardman training simulation system simulates the switching operation, malfunction, and accident process to train grid yardman. After more and more transformer substation has been established, the training demand is enlarged. The early transformer station simulation mechanism is based on real device, after the application of computer technology especially virtual reality technology, 3D virtual device and pure digital substation has ex- 
perienced a rapid development. The integrated system of digital-physical simulation device, central control station and substation has become the mainstream product.

With the adjustment of the substation running management mode, the training simulation system which is suitable for operational integration mode is still blank. As a result, the current training simulation system can't satisfy the training needs of operational skills of talent already. Therefore, developing a set of electric power, comprehensive Simulation Training System which is aimed at training of operation positions, and that will effectively improve the overall level of the training, the teaching of overhaul, training of appraisal of techniques and ways of assessments has vital meanings.

In this paper, based on the research of the existing Simulation Training System, the author developed and successfully implemented operational power mechanic school integration Simulation Training System of the Datong Senior Electrical Engineering School and Jibei Power training center. The SIMULATION TRAINING SYSTEM concludes grids, substations, the paper introduced the system's the function of each part of the training methods, and how to realize an organic combination of implementation. System can not only realize the production unit operation personnel patrol, accident treatment and daily operation, abnormal joint anti-accident exercises training of the power grid and the substation simulation system, but can also realize substation equipment's routine C and D level maintenance and routine test of visual experience [4]. This paper introduces the general structure of the Simulation Training System, and its key technology in the process of system implementation, applications of the system are introduced in the last part of the paper.

\section{General Structure of the System}

The general construct of SIMULATION TRAINING SYSTEM is showed in Figure 1. The Simulation Training System concludes grid simulation, transformer station simulation, dispatch automatic system, C and D level regular recondition simulation, experimental transformer substation regular simulation. Send orders to trainee through teaching system to perform the cooperation of different parts of simulation module. The function of the Simulation Training System is as follow:

1) Grid simulation: transient state, metaphase, long process integrated simulation method, performing Real-time calculation of power grid, express power system dynamic behavior accurately. Use Power flow calculation through simulation operation support system, requiring data distribution for comprehensive Simulation Training System, to guarantee the system operation condition and the consistency of the data.

2) Substation simulation, visualization, modular three-dimensional simulation technology was adopted to realize the substation in the primary and secondary equipment of 3D scenes, virtual equipment perform the right action according to the operation support system, at the same time, students can stay in $3 \mathrm{~d}$ patrol system to carry out patrol and operations on the primary and secondary equipment.

3) Dispatch automation simulation system: to power dispatch system as the prototype, the main simulation monitoring function of SCADA, man-machine interface and operation process.

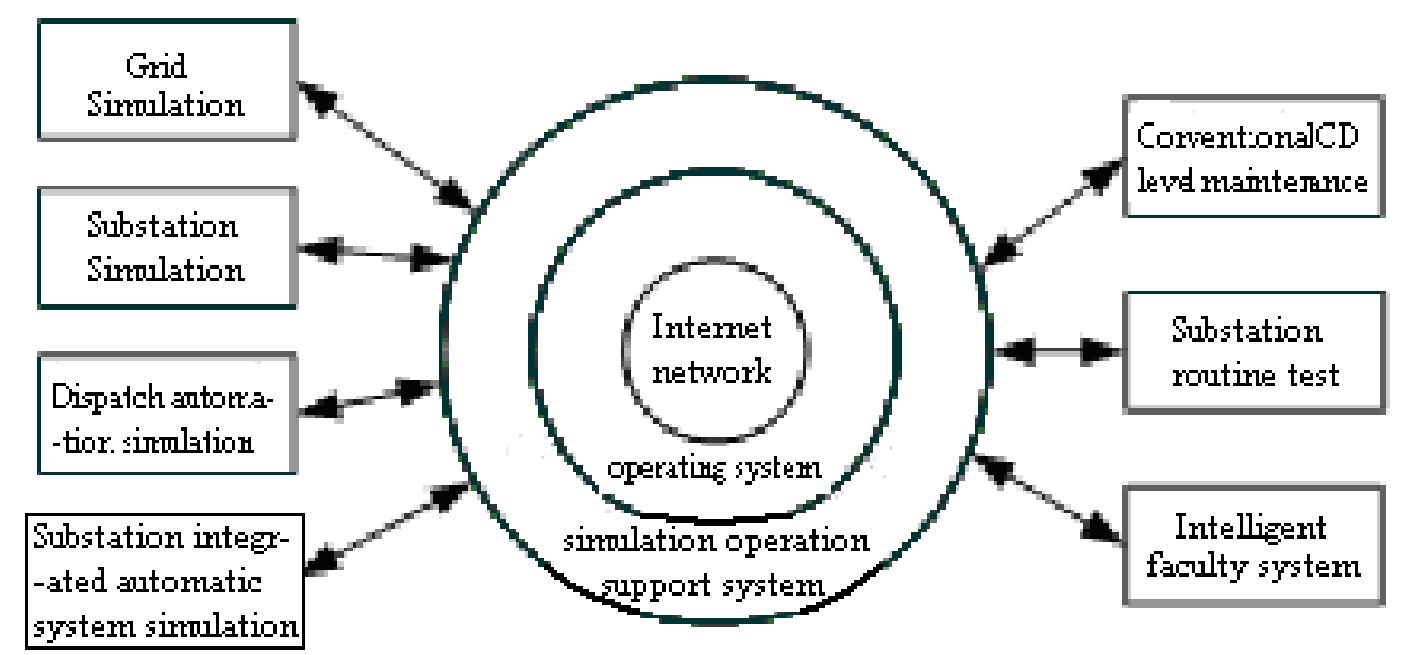

Figure 1. Operational integration simulation training system’s overall structure. 
4) Substation integrated automatic simulation system: the main simulation function of monitoring and control system, man-machine interface and the operation process, provide students with a virtual working environment.

5) The conventional substation simulation of $C$ and $D$ level maintenance: repair work is based on the substation running simulation scenario, to development a better simulation environment for conventional substation maintenance training. Operators can finish all kinds of tasks in this substation routine maintenance scenario.

6) The routine test transformer substation simulation: through the establishment of the visualization of routine test transformer substation simulation scenarios, the system let the trainees practice the basic knowledge of substation routine test, test instrument operation, routine test work processes and other job training, realize the whole dynamic simulation training process of routine test for trained personnel.

7) The faculty system: to realize a unified control to run all of the simulation system on the support platform, teachers send the system state through data system, state data simulation system automatically performs the data distribution management services to all parts of the system.

On the basis of the simulation operation support system, the interaction between each part of the simulation module, construct the integration training simulation system together. After the accident and abnormal in simulation grid, substation equipment will correctly response, grid act correspondingly to changes in trends and to run other simulation module, the module will complete action message alarm information, alarm signal, protection and automatic device according to the protection configuration, combined with the conventional maintenance test.

\section{Key Techniques of the System}

\subsection{Operation Support Platform Based on High Level Architecture (HLA) [5]}

Refer to the international standard IEEE1516 standard series, perform the further research of simulation application management modeling in distributed, interactive power simulation operation support platform's simulation component management, statement management, time management and data distribution management technology, build simulation application support plug and play, which has the function of time collaborative simulation operation support platform.

As shown in Figure 2, the operating support environment is connected through communication soft standard interface to the simulation application. As long as it is based on HLA simulation application standard interface, it can realize plug and play. Operating support environment is the realization of a high level architecture, it fully

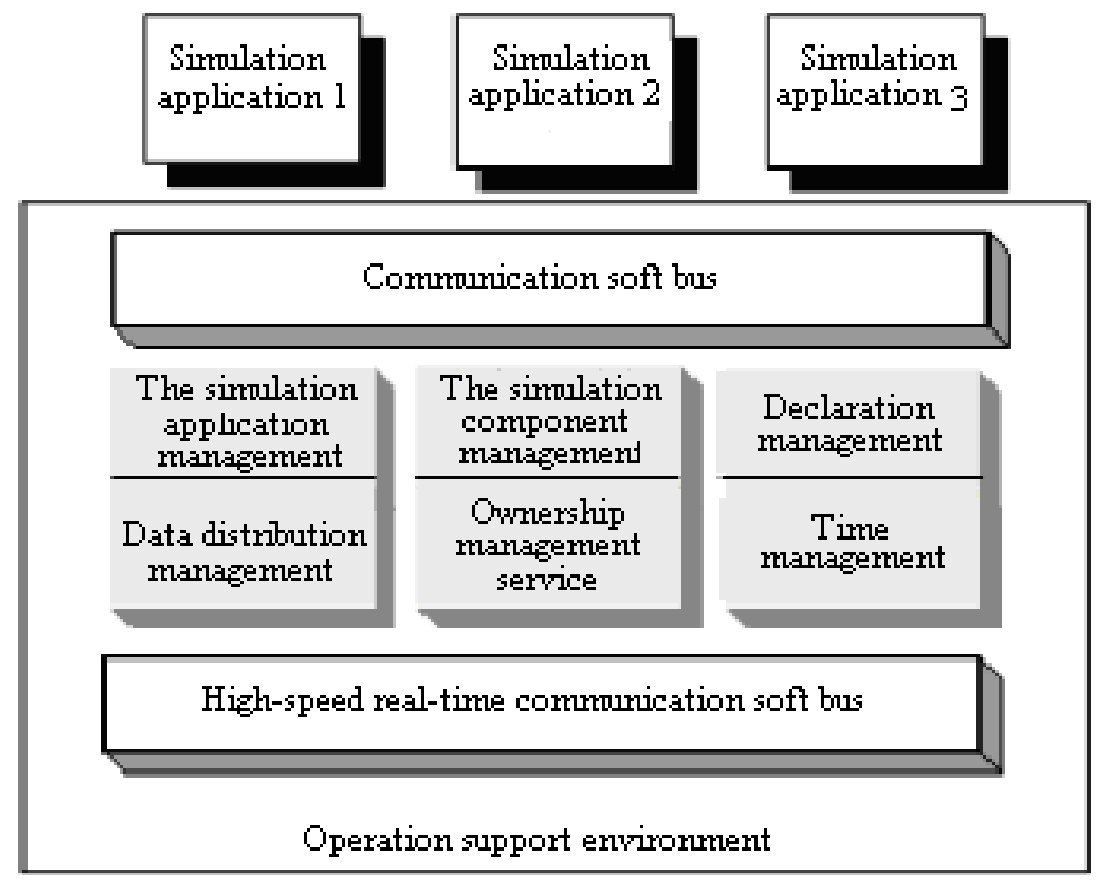

Figure 2. Operation support platform structure. 
follows the high-level architecture specification, to ensure the operation support environment to realize the function through the standardization of technical specifications has nothing to do with specific application system, which concludes the characteristics of generality, openness and easy extension, and realizes the simulation of different types, different time advance mechanism interoperability between applications. Solving the reusability and interoperability of simulation application from the architecture, make it easy to the integration of new simulation system, management and reconstruction, the ideal is the coordination of complex simulation system support environment.

SIMULATION TRAINING SYSTEM is a system contains a variety of different complex simulation, simulation application system requires a variety of simulation application work together. In the aspect of system construction, a system of flexible structure is needed, support system, in accordance with the unified planning and step-by-step implementation continually develops and expands. The operation supports environment based on high level architecture is a good way to meet the needs of the integration of operations simulation system, laid a solid foundation for the success of the system development.

\subsection{Operational Integrated Grid Modeling Technology [6]}

System USES the international and domestic standards (CIM-XML, E language, CIM-E, SVG graphic format, the graph $G$ format) to realize synchronizations with external system model and data interface, in accordance with the training simulation model and application requirements of data integration, to perform the model's calibration, cutting, stitching, equivalence, split, release, and other functions. At the same time, providing visualization and a model of hierarchical modeling tools, to achieve rapid modeling of power grid model. The system provides visual and componentized secondary device modeling tools, implements secondary equipment (equipment, circuit) of modular modeling based on graph model, establishes a foundation for the unification of the secondary equipment and detailed simulation model.

Operational integration training simulation urgently needs to provide a new type of secondary equipment model building technology, on one hand it can accurately describe the detail of the secondary equipment's physical properties of mechanism, on the other hand, it can satisfy the practical requirements of rapid deployment. Based on the definition of IEC61850 standard, the secondary equipment model consists of logical node. As shown in Figure 3, logical node is according to the basic unit of secondary equipment function definition module, logical node contains the corresponding attributes and functions, which can reflect the status of secondary equipment, parameter, setting value, interface and unit connection topology relation, etc.

\subsection{Modular Three-Dimensional Modeling Technology}

3D modeling system uses a visualization, modularization and gallery module integration modeling technology, using the personnel component generator of 3D modeling to edit the 3D model of original equipment, and derived for the three dimensional components can be assembled from the three-dimensional equipment after editing. Component assembler derived by using the component generator of three dimensional components, is convenient to assemble equipment components which can be ran directly by primary and secondary simulation of

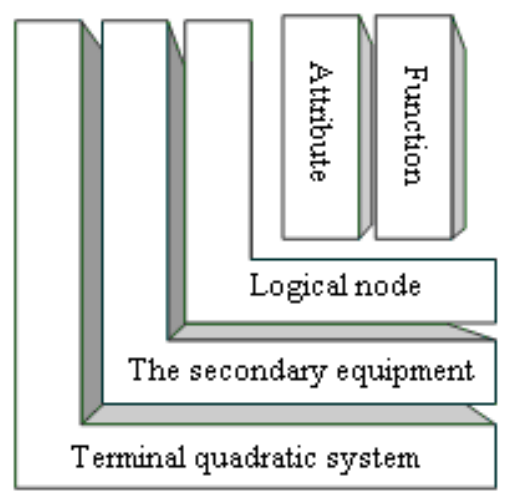

Figure 3. Secondary device simulation model structure. 
3D program. Scene assemblers assembly equipment for 3D simulation training scenario through visual edit mode component, all kinds of equipment components can be quickly assembled for the power of 3D virtual scene, the visualization of the interface assembly can be fully realized by the assembly process, we only need simple mouse operation. Scene assemblers provide a friendly environment, through the mouse drag equipment, components can easily move to the right place, to facilitate the observation scene, realized the function of comprehensive roaming observation, the scene of assembly process can be viewed from various angles to ensure the correct assembly of the scene.

\subsection{Interactive 3D Engine Technology Based on Open GL}

OpenGL 3D graphics based on cross-platform language, develop a set of 3D graphics support engine, which provides a framework based on OpenGL's object-oriented technology, realizes the key techniques and algorithms of 3D engine efficiency and performance, encapsulated the realistic graphics, 3D scene management, sound management, collision detection, object interaction and real time maintenance function, developed a 3D scene interface for a quick and efficient development, the engine considered the characteristics of the power system. To organize the characteristic of scenario with the electrical connection in the scene tree, developers only need to use less code to set the scene for each node of the tree and achieve efficient rendering 3D scene.

3D engine implements the 3D geographic information system data and data scheduling, in view of the massive three-dimensional geographic information data, using the rendering such as tiles segmentation and dynamic scheduling strategy, solving the system operation performance degradation caused by the massive terrain data. $3 \mathrm{D}$ engine implements the satellite remote sensing image and the fusion of 3D terrain data to improve the sense of reality of the system.

\subsection{Substation Maintenance Simulation and Experiment Based on the Technology of Direct X Gaming}

Direct $\mathrm{X}$ is a kind of application program interface, it allows higher execution efficiency for Windows platform game program and strengthen the 3D graphics and sound effects. DirectX is made up by many components: DirectSound: provides voice mixing and playback of hardware and software; Direct3D: a 3 d graphics package which provides an advanced Retained Mode (Retained Mode) interface, this enables the user to achieve a complete 3D graphics system. It also contains a low-level Immediate Mode interface, allows application programs to gain complete control over the rendering pipeline.

Based on the technology of Direct $\mathrm{X}$ gaming, substation maintenance and test the simulation from the interface style, operation mode are similar to common real-time strategy game, trainees in substation virtual scene, select tools and several instruments to finish the maintenance and testing tasks. The use and maintenance of a variety of tools, instruments, experimental steps and the process are exactly the same with their actual characteristics. Authenticity, target, high antagonism, task, help and evaluation, makes the substation maintenance and test the knowledge more easily.

\subsection{Operational Integration Technology of Remote Simulation Training}

Operational integration simulation training of each subsystem can run smoothly in the online electric power enterprises, remote training around the classroom is distributed in the training center, the classroom is equipped with each training, student platform, switches and network equipment, using a VPN network technology can realize a smooth connection between faculty and students platform, perfectly meet the training needs (Figure 4).

Operational integration Simulation Training System has the network buffer server, realizing the simulation timing intelligent backtracking technique and the automatic synchronization of simulation application in remote simulation system. During the broken network automatic reconnection of the training system, the simulation system can adjust the clock rate of intelligent simulation model, with history simulation event and state, to realize the stable synchronization of monitoring center, substation and power grid simulation application in complex network environment.

Operational integration simulation training realized remote network oriented training simulation application mode. The remote user can log into the Simulation Training System, the automaticly download, install, and start the remote training simulation system. 


\section{Application of the System}

Comprehensive operation-maintenances Simulation Training System has realized the integration of power grid, substations, dispatching automation, conventional C, D level maintenance and routine test joint simulation, training normal operation skills for related production personnel, abnormal and accident treatment skills, the related personnel of the C, D level maintenance and routine test. The system has been put into use in Datong Senior Electrical Engineering School and Jibei Power training center, the system overall structure is shown in Figure 5.

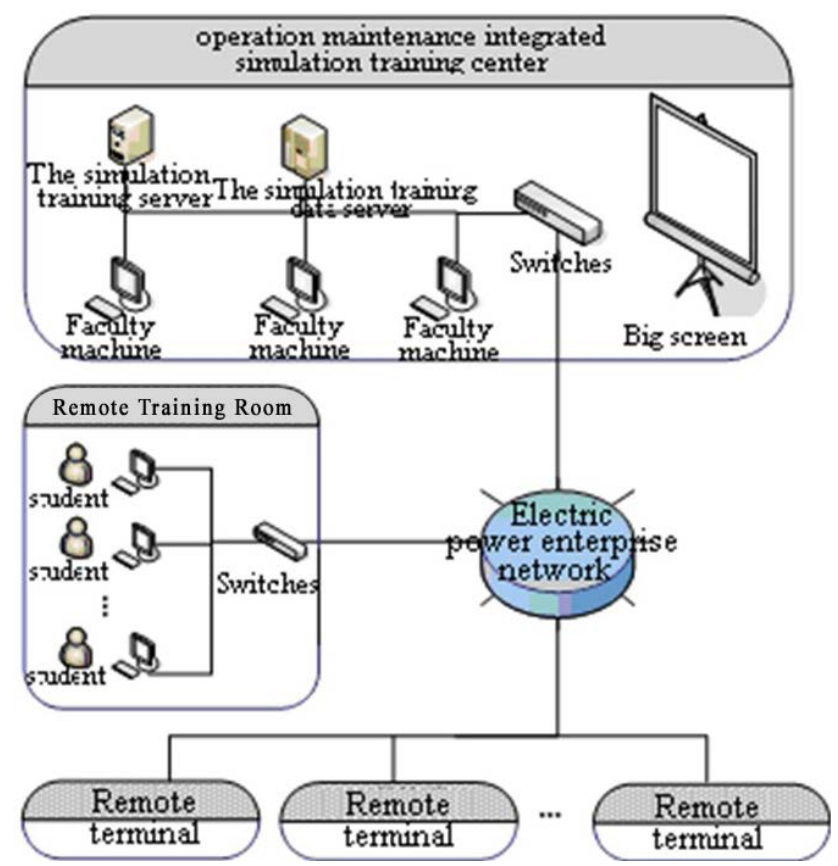

Figure 4. Remote training operations integration.

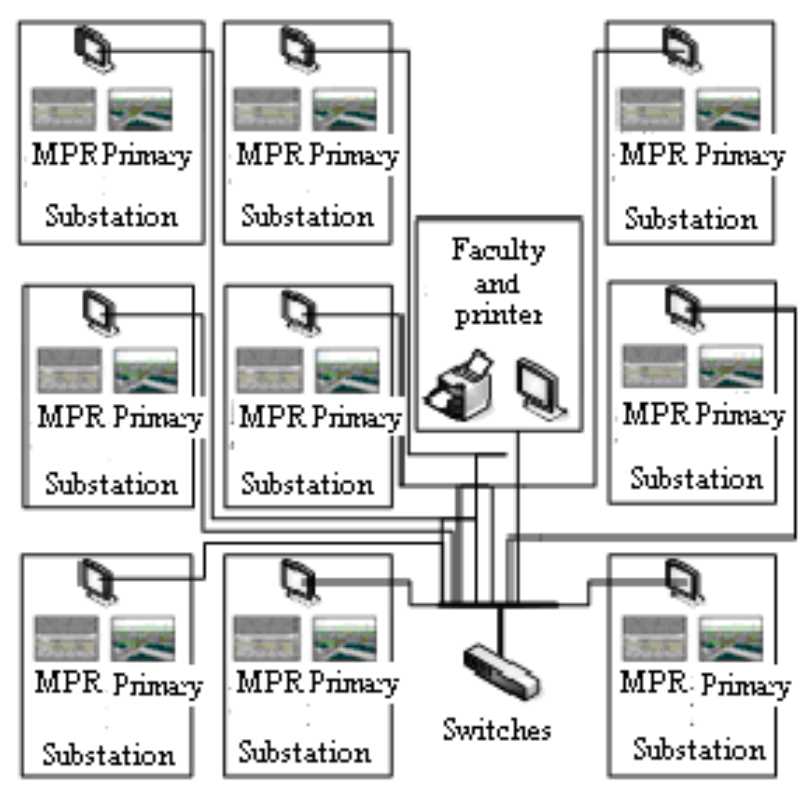

MPR: Monitoring and protection room

Figure 5. Integrated training simulation system overall structure. 
Datong Senior Electrical Engineering School using this system for 8 period, about 240 times of training for substation operations. Students generally praised the value of this system, especially in the substation maintenance part, filled the gaps in simulation training. The gaming interface operation is vivid, simple, practical, and the simulation system trained the trainees how to execute the primary and secondary transformer substation's $\mathrm{C}$ and D level maintenance, improved their professional skills.

\section{Conclusion}

Simulation Training System is a set of joint operations integration Simulation Training Systems, add high level architecture, virtual reality technology, the technology of Direct X, substation C, D level maintenance and routine test, on the basis of the original grid, substation simulation, solves the problem of lacking maintenance, build an actual integrated operation management mode for the first time. The whole process of the digital simulation of the environment and the whole scene, covers the relevant operational integration production operation personnel's training, examination, appraisal and joint anti-accident exercises and other functions, which can effectively improve grid operational integration professional skills to improve production efficiency, shorten the training cycle, improve the quality of training, and foster a staff team which meets the needs of "overhaul" system, rich professional knowledge, operation skill skilled high-quality in a relatively short period of time, ensuring the safety of power grid stability, economic efficient operation, well prepared for a grid production management mode under overhaul system, to meet China's “overhaul” power grid’s needs in the future.

\section{References}

[1] Zhang, Q. After the "Big Five” Three Reforms How to Deepen the Staff Skill Training Work. Science and Technology Innovation and Application, $\mathbf{3 0 .}$

[2] Chen, X.Z. and Hao, Y.C. (2011) Overhaul Organization Integration of Operational Personnel Training Research. Electric Power Education in China, 9.

[3] Guo, W. (2013) Substation Operations Integration Scheme and the Existing Problems and Solutions. Computer Engineering Application Technology, 2.

[4] Xu, Y.Y. (2012) Substation Operational Scheme Are Discussed. China Power Education, 33.

[5] Yang, X.H., Lin, C., Kingdoms Equality (2009) Based on High Level Architecture Integrated Simulation Platform of Power System. Power Grid Technology, 11, 98-103.

[6] Bai, A.M., Xu, Z.Q. and Tan, L. (2012) Grid Control Integration of Research and Application of Simulation Training System Development Strategy. Qinghai Electric Power, 1. 